\title{
1 High energy supercapattery with an ionic liquid solution of $\mathrm{LiClO}_{4}$
}

2 Linpo $\mathrm{Yu}^{\mathrm{a}, \mathrm{b}}$ and George Z. Chen ${ }^{\mathrm{a}, \mathrm{b}_{*}}$

a Department of Chemical and Environmental Engineering, and Energy and Sustainability

4 Research Division, the University of Nottingham, Nottingham, NG7 2RD UK

Email: George.Chen@nottingham.ac.uk

${ }^{b}$ Department of Chemical and Environmental Engineering, and Centre for Sustainable Energy

Technologies, Faculty of Science and Engineering, the University of Nottingham Ningbo China,

$8 \quad$ Ningbo, 315100 China

9

\section{Abstract:}

Supercapattery combining an ideally polarized capacitor-like electrode and a battery-like electrode is demonstrated theoretically and practically using an ionic liquid electrolyte containing 1-butyl-1-methylpyrrolidinium tri(pentafluoroethyl)trifluorophosphate (BMPyrrFAP), gamma-butyrolactone $(\gamma-\mathrm{GBL})$ and $\mathrm{LiClO}_{4}$. The electrochemical deposition and dissolution of lithium metal on a platinum and glass carbon electrode were investigated in this ionic liquid solution. The $\mathrm{CV}$ data shows the fresh electrochemical deposited lithium metal is stable in the electrolyte, which encourages the investigation of this ionic liquid solution in supercapattery with a lithium battery negative electrode. The active material counted specific energy of the supercapattery based on a lithium negative electrode and an activated carbon (Act-C) positive electrode can reach $230 \mathrm{Wh} \mathrm{kg}^{-1}$ under the Galvanostatic charge-discharge current density of 1 $\mathrm{mA} \mathrm{cm}{ }^{-2}$. The positive electrode material, Act-C was also investigated by $\mathrm{CV}, \mathrm{AC}$ impedance, SEM and BET. The non-uniform particle size and micropore porous structure of the Act-C enable its electric double layer capacitor (EDLC) behavior in the ionic liquid solution. The 
1 calculated specific capacitance of the Act-C in this ionic liquid solution is higher than same Act-

$2 \mathrm{C}$ in aqueous solution, which indicates the pseudocapacitance behaviour of Act- $\mathrm{C}$ with the 3 species in the ionic liquid electrolyte.

\section{Introduction}

6 Supercapattery (=supercapacitor + battery) takes the advantages of both supercapacitor

7 (also known as electric double layer capacitor, or EDLC) and battery by combining an ideally

8 polarized capacitor-like electrode and a battery-like electrode. ${ }^{1}$ Although lithium ion capacitor ${ }^{2,3}$

9 is also comprised of this hybrid configuration, supercapattery is the more general term of the 10 particular design.

11 In theory, supercapattery can possess higher energy density than both battery and 12 supercapacitor and can supply this energy at a power output almost as high as supercapacitor. 13 The high power output of supercapattery is mainly a result of sharing the same electrochemical 14 active materials with supercapacitor, where the nanostructured carbons, like activated carbon 15 (Act-C), carbon nanotubes (CNTs) and graphene are the best choice for the ideally polarized 16 electrode because of their large surface area, porosity, stability over a wide potential window, 17 and intrinsically low electrical resistance. In addition, pseudo-capacitive materials including $18 \mathrm{MnO}_{2}, \mathrm{RuO}_{2}$ and conducting polymers can also be used as the capacitor-like electrode materials 19 providing the high electrode capacitance, but limiting the potential windows.

As to the battery-like electrode, various electrode materials from commercial battery 21 systems can be the candidate, from lead acid batteries to metal/air systems, but in practice, metal 22 compounds ${ }^{3-5}$ like $\mathrm{SnO}_{2}, \mathrm{MnO}_{2}$ and $\mathrm{LiFePO}_{4}$ are more common and commercially available. 23 Theoretically, the hypothetical battery comprising lithium metal and fluorine gas (Li-F battery) 
1 would output a cell voltage about $6.1 \mathrm{~V}$ and offer a specific energy content of $6304 \mathrm{Wh} \mathrm{kg}^{-1}$. Any

2 battery cannot go beyond the specific energy content of the hypothetical Li-F battery. As to Li-

3 ion battery $\left(\mathrm{Li}_{\mathrm{x}} \mathrm{C}_{6} \mid \mathrm{Li}_{1-\mathrm{x}} \mathrm{CoO}_{2}\right)$, the theoretical specific energy is $552 \mathrm{Wh} \mathrm{kg}^{-1}$ at $3.5 \mathrm{~V}$. A

4 hypothetical supercapattery comprising a lithium metal negative electrode and a supercapacitor

5 positive electrode (assumed $400 \mathrm{~F} \mathrm{~g}^{-1}$ ) is evaluated and analyzed here. Considering the specific

6 charge capacity of lithium is much larger than that of the supercapacitor electrode, the mass of

7 the lithium metal is negligible. The theoretical specific energy value would be $625 \mathrm{Wh} \mathrm{kg}^{-1}$ for

8 the cell voltage vary from $3.5 \mathrm{~V}$ to $1.0 \mathrm{~V}$. This theoretical value is even higher than the one of

9 the Li-ion battery.

$11 \quad E_{\max }=\frac{1}{2} C U_{\max }^{2}$

12 where $C$ is the specific capacitance of a capacitor, and $E_{\max }$ is the maximum energy capacity of a capacitor correlated to its maximum tolerable voltage, $U_{\max }$. It should be mentioned that a hybrid

14 cell of a battery electrode and a supercapacitor electrode does not always show typical supercapacitor behaviour, where the cell voltage $(U)$ is always proportional to the time $(\mathrm{t})$ during 16 a constant current charging or discharging test. If the cell presents battery like features, the term 17 supercabattery is recommended, ${ }^{6}$ but not discussed in this paper. In the case of supercapattery, 18 the $U_{\max }$ is a key factor for the energy capacity of the devices. Because the behaviour of 19 supercapattery is close to that of supercapacitor, ${ }^{1}$ several strategies that have been applied in supercapacitor can be utilized in supercapattery to improve the practical energy capacity, such as

21 using the design of asymmetric supercapacitor cell, ${ }^{7}$ controlling the capacitance ratio of the 22 positive and negative electrode made from the same material, ${ }^{8}$ and serially stacking the cells 23 through the bipolar electrodes. ${ }^{9}$ Apart from these efforts based on the aqueous electrolytes, there 
1 is a strong desire for changing the aqueous to organic electrolytes to achieve a high working

2 voltage.

3 Recent studies have revealed both aqueous and non-aqueous supercapatteries using lithium

4 metal $^{10}$ and Li-ion battery material ${ }^{3}$ as the electrode. An aqueous supercapattery consisting of a

$5 \mathrm{MnO}_{2}$ positive electrode and a Li/LISICON/PEO-LiTFSI/Li ${ }^{+}$negative electrode had achieved a

6 specific energy capacity of $114 \mathrm{Wh} \mathrm{kg}^{-1}$ with a $4.3 \mathrm{~V}$ cell voltage. ${ }^{10}$ The Li/LISICON/PEO-

$7 \mathrm{LiTFSI} / \mathrm{Li}^{+}$electrode is a multi-layered $\mathrm{Li}$ electrode, which consists of lithium metal, a

8 LISICON-type solid glass ceramic as the water-stable solid electrolyte, and a buffer layer

9 consisting of polyethylene oxide with $\mathrm{Li}\left(\mathrm{CF}_{3} \mathrm{SO}_{2}\right)_{2} \mathrm{~N}$ polymer electrolyte (PEO-LiTFSI) between

10 the lithium metal and the solid electrolyte. If $\mathrm{MnO}_{2}$ is replaced by $\mathrm{RuO}_{2}$ as the positive electrode,

11 the specific energy capacity of the device comes to $520 \mathrm{Wh} \mathrm{kg}^{-1}$ with a $3.8 \mathrm{~V}$ cell voltage. ${ }^{10}$

12 However, the current density of the aforementioned devices is only $0.255 \mathrm{~mA} \mathrm{~cm}{ }^{-2}$, which is

13 limited by the solid/liquid interphase. This disadvantage prevents these high energy capacity

14 devices in any high power application. Another non-aqueous supercapattery using $\mathrm{LiFePO}_{4}$ as

15 the positive electrode and Cabot carbon black as the negative electrode possesses good cycling

16 stability at high current density in a Li-ion contained propylene carbonate (PC) electrolyte. ${ }^{3}$

17 However, the potential range of the Cabot carbon black can only keep its capacitor-like

18 behaviour from 2.80 to $1.25 \mathrm{~V} \mathrm{vs.} \mathrm{Li} / \mathrm{Li}^{+}$in the electrolyte, which has limited the cell voltage.

Ionic liquids are specially featured by their zero or negligible volatility, but still able to offer

20 the highly ionic environment, and wide temperature and potential windows. ${ }^{11}$ They have brought

21 about unique opportunities, including synthesis, ${ }^{12}$ trace analysis, ${ }^{13,14}$ thermochromic/cryochromic

22 materials, ${ }^{15-17}$ and electrochemical energy storage. ${ }^{18-20}$ Consequently, ionic liquid solutions can 
1 be chosen as competitive candidates for the electrolytes of supercapattery when the battery-

2 behaviour electrode is a lithium-ion or lithium battery negative electrode.

3 Here, we report the recent supercapattery work based on an activated carbon positive

4 electrode and a $\mathrm{Li} / \mathrm{Li}^{+}$negative electrode using an ionic liquid electrolyte, 1-butyl-1-

5 methylpyrrolidinium tri(pentafluoroethyl)trifluorophosphate (BMPyrrFAP) containing gamma-

6 butyrolactone $(\gamma-\mathrm{GBL})$ and $\mathrm{LiClO}_{4}$. The characterization of the activated carbon has been done

7 and presented here, including the data of cyclic voltammogram (CV), AC impedance, SEM and

8 BET. The demonstrated supercapattery cell shows a clear capacitor-like behaviour in the CV and

9 Galvanostatic charge and discharge (GCD) tests. This particular hybrid design ensures the full

10 usage of the electrochemical window of the ionic liquid solution and the capacitance of the

11 activated carbon, and basically maintains the high power of the supercapacitor.

\section{Experimental}

In this work, a supercapacitor grade commercial product of activated carbon (Act-C, YP50F,

15 Kuraray Chemical Co.) was used. The other chemicals, 1-butyl-1-methylpyrrolidinium 16 tri(pentafluoroethyl)trifluorophosphate (BMPyrrFAP, Merck), 1-ethyl-3-methylimidazolium

17 tetracyanoborate (EMIM[B(CN) $\left.)_{4}\right]$, Merck), gamma-butyrolactone ( $\gamma$-GBL, Sigma Aldrich) and $18 \mathrm{LiClO}_{4}$ (Sigma Aldrich), were commercially available and used without further purification. 19 Lithium metal (foil, Sigma Aldrich) was kept and handled in an argon filled glove box. The 20 homemade electrochemical cells (2-electrode/3-electrode cells and sandwich type cell) were 21 fabricated in an argon-filled glove box $\left(\mathrm{O}_{2}<10 \mathrm{ppm}, \mathrm{H}_{2} \mathrm{O}<10 \mathrm{ppm}\right)$ and transferred outside the 22 glove box for the electrochemical experiments by an AUTOLAB $302 \mathrm{~N}$ potentiostat. Membrane 23 from Celgard was used as the separator in the sandwich type cell. 
For the electrochemical tests, the Act-C powder was made into pellet type and casted electrodes with PTFE and PVDF, respectively. The details of fabricating the Act-C/PTFE pellet electrode can be found in previous publication from this laboratory. ${ }^{21}$ The Act-C/PVDF electrode was fabricated by the following process. $10 \mu \mathrm{L}$ of an Act-C suspension $(0.950 \mathrm{~g}$ Kuraray Act-C and $0.050 \mathrm{~g}$ PVDF powder in $20 \mathrm{~mL}$ DMSO suspension) was dropped on a $5 \mathrm{~mm}$ diameter graphite disc electrode. The electrode loaded with $0.5 \mathrm{mg}$ of Act-C composite $(95 \% \mathrm{w}$. Act-C and $5 \% \mathrm{w}$. PVDF) was dried in a vacuum oven at $75{ }^{\circ} \mathrm{C}$ overnight, and then was ready for the electrochemical tests.

All the experiment is operated at room temperature. More experimental details are specified in the following sections.

\section{Results and discussion}

The pore size and volume distribution of an Act-C are the most important physical characteristics. Fig. 1 presents the SEM images of an Act-C pellet containing $5 \%$ w. PTFE. The Act-C powder is non-uniform in particle size, and the PTFE is binding the Act-C particles like a spider web to maintain the mechanical strength of the Act-C pellet. The surface area of the Kuraray Act-C sample is $1724 \mathrm{~m}^{2} \mathrm{~g}^{-1}$ determined from the nitrogen absorption/desorption isotherms at $77 \mathrm{~K}$ (ASAP 2420) by applying the density function theory (DFT). In previous work, ${ }^{21}$ the dominant range of pore widths of the same Kuraray Act-C sample is between 1.5 and $2.5 \mathrm{~nm}$. For an aqueous electrolyte, its wetting ability with the Act-C pellet will affect the contact between the electrolyte and Act-C particles, and vary the charge storage performance of the ActC sample. As to the organic electrolyte, the wetting is not a problem anymore, while the interaction between electrolyte and carbon materials inside the pore will play an important role. 
1 A theory was proposed for the traditional organic electrolyte, that the longer the pore inside the

2 particle is, the poorer performance of the capacitance of the porous carbon materials is. ${ }^{3}$ This is

3 mainly due to the ion block of the pores by the non-ionic solvent molecular. Similar phenomenon

4 was also observed in the case of aqueous electrolyte with organic additive. ${ }^{21}$

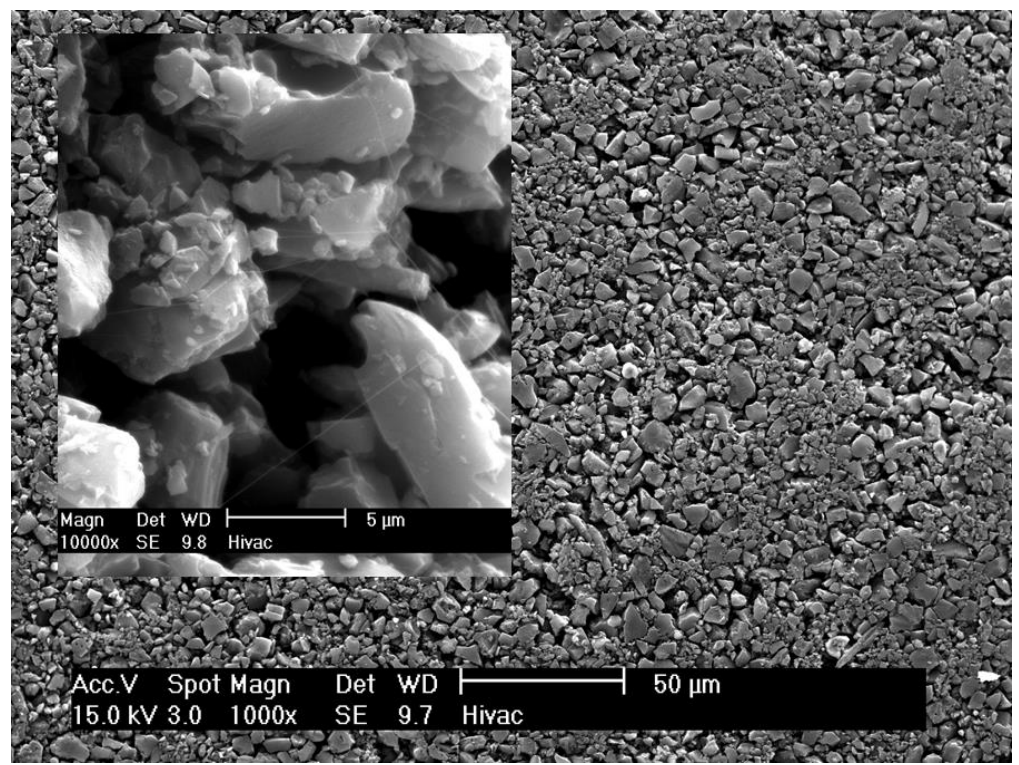

Fig. 1 SEM images of the activated carbon pellet (including $5 \% \mathrm{w}$. PTFE). The insert is the enlarge view of the back image.

Before the work on the supercapattery, we have investigated the Kuraray Act-C in a commercial ionic liquid, 1-ethyl-3-methylimidazolium tetracyanoborate $\left(\operatorname{EMIM}\left[\mathrm{B}(\mathrm{CN})_{4}\right]\right)$. Fig. 2 shows the photo of electrochemical tube cell and test results on CV, GCD and AC impedance. A small piece of Act-C pellet (about $1 \mathrm{mg}$, containing $5 \% \mathrm{w}$. PTFE) was loaded on a $6 \mathrm{~mm}$ diameter graphite disc electrode. Another graphite disc attaching $30 \mathrm{mg}$ Act-C was used as the counter electrode. Reference electrode is a $\mathrm{Ag} / \mathrm{Ag}^{+}$electrode filled with $0.01 \mathrm{~mol} \mathrm{~L}^{-1} \mathrm{AgNO}_{3}$ acetonitrile solution, which is connected to the electrolyte via a salt bridge filled with EMIM $\left[\mathrm{B}(\mathrm{CN})_{4}\right]$. This reference can be prepared following the method described in reference. ${ }^{22}$ It 
1 should be mentioned that the reference electrode is changed to $\mathrm{Li} / \mathrm{Li}^{+}$electrode in the latter

2 discussion except in Fig. 2. Any potential in this paper will be suffixed with the reference

3 electrode used in the experiment.
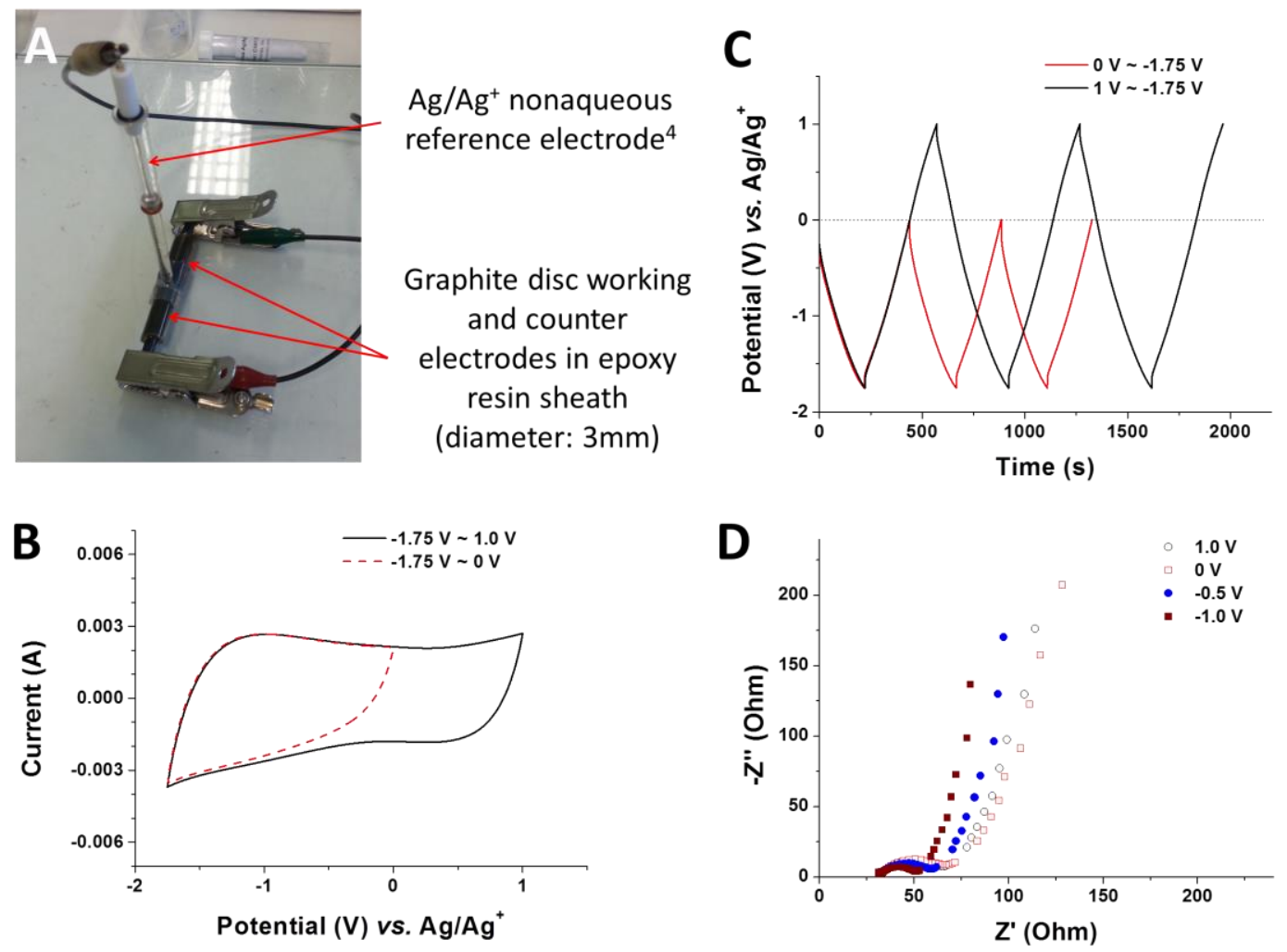

5 Fig. 2 (A): Photograph of the tube cell. $1.064 \mathrm{mg}$ Act-C (including 5\% w. PTFE) was loaded on

6 the graphite disc as working electrode. $\operatorname{EMIM}\left[\mathrm{B}(\mathrm{CN})_{4}\right]$ was filled in the tube as electrolyte.

7 Reference electrode is $\mathrm{Ag} / \mathrm{Ag}^{+}$electrode in $0.01 \mathrm{~mol} \mathrm{~L}{ }^{-1} \mathrm{AgNO}_{3}$ acetonitrile solution connected 8 to the electrolyte via a salt bridge filled only with $\operatorname{EMIM}\left[\mathrm{B}(\mathrm{CN})_{4}\right]$. The results in $(\mathbf{B}),(\mathbf{C})$ and

9 (D) were collected from this tube cell.

10 (B): CVs of the tube cell. Scan rate: $20 \mathrm{mV} \mathrm{s}^{-1}$.

11 (C): Galvanostatic Charge-Discharge plots of the tube cell. Current: $1 \mathrm{~mA}$.

12 (D): Nyquist plots of the tube cell at potentials indicated in the figure. 
Both CVs and AC impedance results in Fig. 2 indicate that the Kuraray Act-C doesn't show

2 a pure EDLC performance in $\operatorname{EMIM}\left[\mathrm{B}(\mathrm{CN})_{4}\right]$. The Act-C can store more charge in a potential

3 range from -1.7 to $0 \mathrm{~V}$ vs. $\mathrm{Ag} / \mathrm{Ag}^{+}$than the range of 0 to $1.0 \mathrm{~V}$ vs. $\mathrm{Ag} / \mathrm{Ag}^{+}$. If a symmetrical

4 supercapacitor was fabricated using this Act-C and ionic liquid, the maximum voltage could not

5 go beyond $2.7 \mathrm{~V}$. The calculated specific capacitance value of Kuraray Act-C in EMIM[B $\left.(\mathrm{CN})_{4}\right]$

6 is about $100 \mathrm{~F} \mathrm{~g}^{-1}$, which is comparable to the value of the same sample got in aqueous

7 solution. $^{21}$ The voltage of this ionic liquid based symmetrical cell is not increased dramatically,

8 while the resistance of the cell will increased compared to the cell using an aqueous electrolyte.

9 In this case, changing electrolyte from an aqueous to a non-aqueous electrolyte is not economic.

10 A new strategy should be made to improve the energy capacity of the cell.

11 As aforementioned, the lithium battery or Li-ion battery processes very high energy capacity

12 mainly caused by the very negative potential of $\mathrm{Li} / \mathrm{Li}^{+}$or relevant redox couple. We chose an

13 ionic liquid which was used as Li-ion battery electrolyte in the following work. The ionic liquid

14 solution containing $\mathrm{LiClO}_{4}$ was used as the electrolyte of the supercapattery. The lithium salt is

15 the Li-ion source for the electrochemical deposition and dissolution of lithium metal on the

16 negative electrode of the supercapattery.

17 Fig. 3A and 3B shows clearly the electrochemical deposition and dissolution of lithium in

18 the ionic liquid electrolyte containing lithium salt on a $2 \mathrm{~mm}$ diameter $\mathrm{Pt}$ disc electrode and a 3

19 mm diameter Glass Carbon (GC) disc electrode, respectively. Fig $2 \mathrm{C}$ is the photo of the

20 homemade electrochemical cell, in which a working electrode (Pt or GC) and a piece of lithium

21 metal foil as both counter and reference electrodes are sealed in a vial. The electrolyte contains

$220.01 \mathrm{~mol} \mathrm{~L}^{-1} \mathrm{LiClO}_{4}$ in the mixture of BMPyrrFAP and $\gamma$-GBL (volume ratio 1:1). Lithium metal

23 can be electrochemically deposited on both substrate materials, and the fresh deposited lithium is 
1 stable in the electrolyte unless a positive scan occurs showing a dissolution peak in the CVs. The

2 over-potential on the GC electrode indicates an energy gap between condense carbon and lithium

3 surface. Another key information from Fig. 3 is that the ionic liquid solution used in this work

4 shows an electrochemical window more than $4 \mathrm{~V}$ using Pt and GC electrodes. The ionic liquid 5 solution could be a potential candidate for the supercapattery electrolyte.
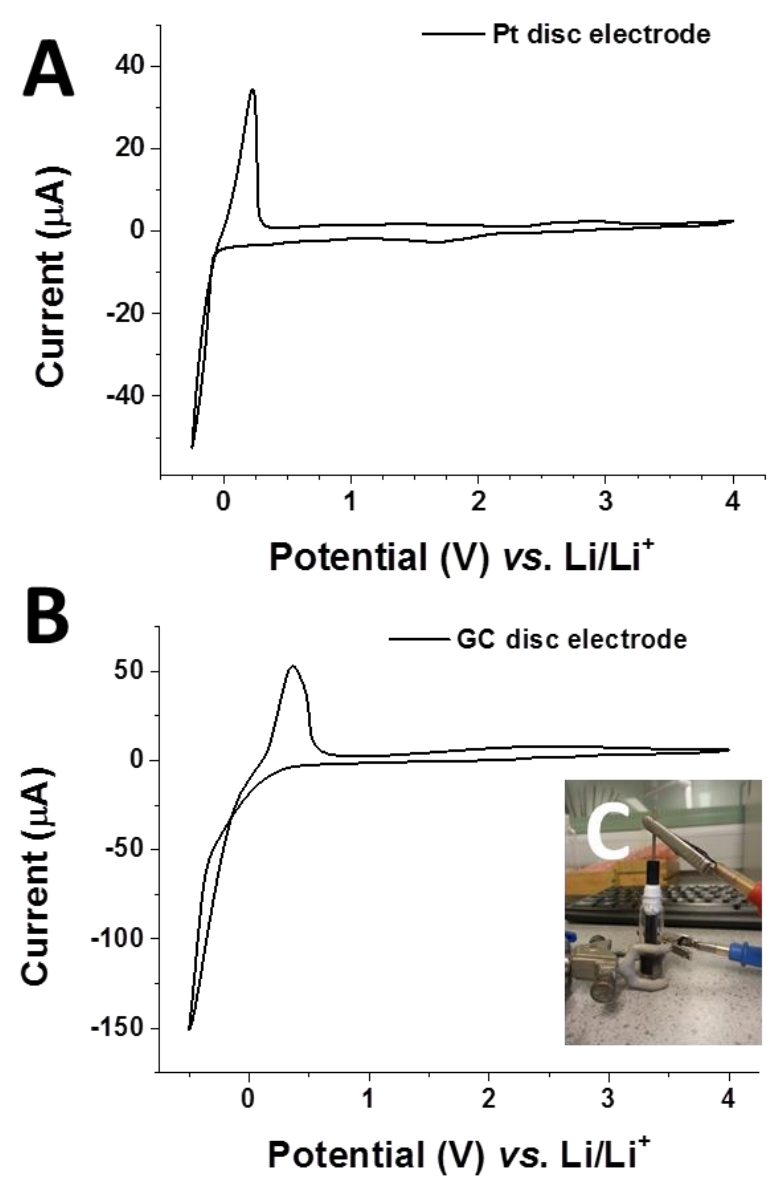

7 Fig. $3 \mathrm{CVs}$ collected on (A) a $2 \mathrm{~mm}$ diameter Pt disc electrode and (B) a $3 \mathrm{~mm}$ diameter Glass

8 Carbon disc electrode, in the mixture of BMPyrrFAP and $\gamma$-GBL (v:v = 1:1), contains 0.01 mol

$9 \mathrm{~L}^{-1} \mathrm{LiClO}_{4}$. Scan rate $10 \mathrm{mV} \mathrm{s}^{-1}$. Potential range: $-0.25 \sim 4.00 \mathrm{~V}$ vs. $\mathrm{Li} / \mathrm{Li}^{+}$. (C) Photo of the 10 homemade electrochemical cell. 
In a demonstrative supercapattery cell with lithium metal negative electrode, the redox

$2 \mathrm{Li} / \mathrm{Li}^{+}$reaction should be occurring during the charging or discharging process. A $0.5 \mathrm{~mol} \mathrm{~L}{ }^{-1}$

$3 \mathrm{LiClO}_{4}$ ionic liquid solution was prepared as the electrolyte to provide sufficient $\mathrm{Li}$-ion in the

4 electrolyte. The solvent content is the same as the one in the previous lithium deposition

5 experiment, BMPyrrFAP and $\gamma$-GBL $(\mathrm{v}: \mathrm{v}=1: 1)$. The working electrode is a piece of $0.5 \mathrm{mg}$

6 Kuraray Act-C pellet coated on a $5 \mathrm{~mm}$ diameter graphite disc electrode with PVDF binder. The

7 counter and referenced electrodes are lithium metal foil. The cell was fabricated in an argon

8 filled glove box, sealed properly and then transferred outside the box for the further test. The CV

9 and AC impedance data of this cell is presented in Fig. 4. The blue dash lines on CV curve mark

10 the potential at which the AC impedance test was run. It should be mentioned again that the real

11 amount of lithium metal anticipating the charge storage process is negligible according the

12 previous calculation. The reason of placing a piece of lithium metal foil in the cell is to acquire

13 accurate potential result during the charging and discharging processes. From the CV in Fig. 4,

14 we found that the Kuraray Act-C can undergo a capacitor-like behaviour from the potential range

15 from 1.7 to $4.3 \mathrm{~V}$ vs. $\mathrm{Li} / \mathrm{Li}^{+}$at least. There is no obvious turning point to the clear oxidative or

16 reductive current on the $\mathrm{CV}$ curve. If we assumed this is capacitor behaviour, the calculated

17 specific capacitance value is $101 \mathrm{~F} \mathrm{~g}^{-1}$. The deformed rectangular $\mathrm{CV}$ shape should be caused by

18 the resistance of the whole cell, which is clearly shown in the Nyquist plots of the cell at

19 different potentials. Although the resistance of the cell shows a value about $180 \Omega$, the

20 equivalent series resistance (ESR) is about $35 \Omega \mathrm{cm}^{2}$ (area: $0.196 \mathrm{~cm}^{2}$ ), which is only 30 times

21 larger than the value got from a symmetrical aqueous AC supercapacitor using the same

22 electrode fabricating method. ${ }^{21}$ In this case, the resistance as shown in Fig. 4 still falls in the 
1 acceptable range in practical devices. The optimization of this hybrid design is definitely needed

2 to increase output power.
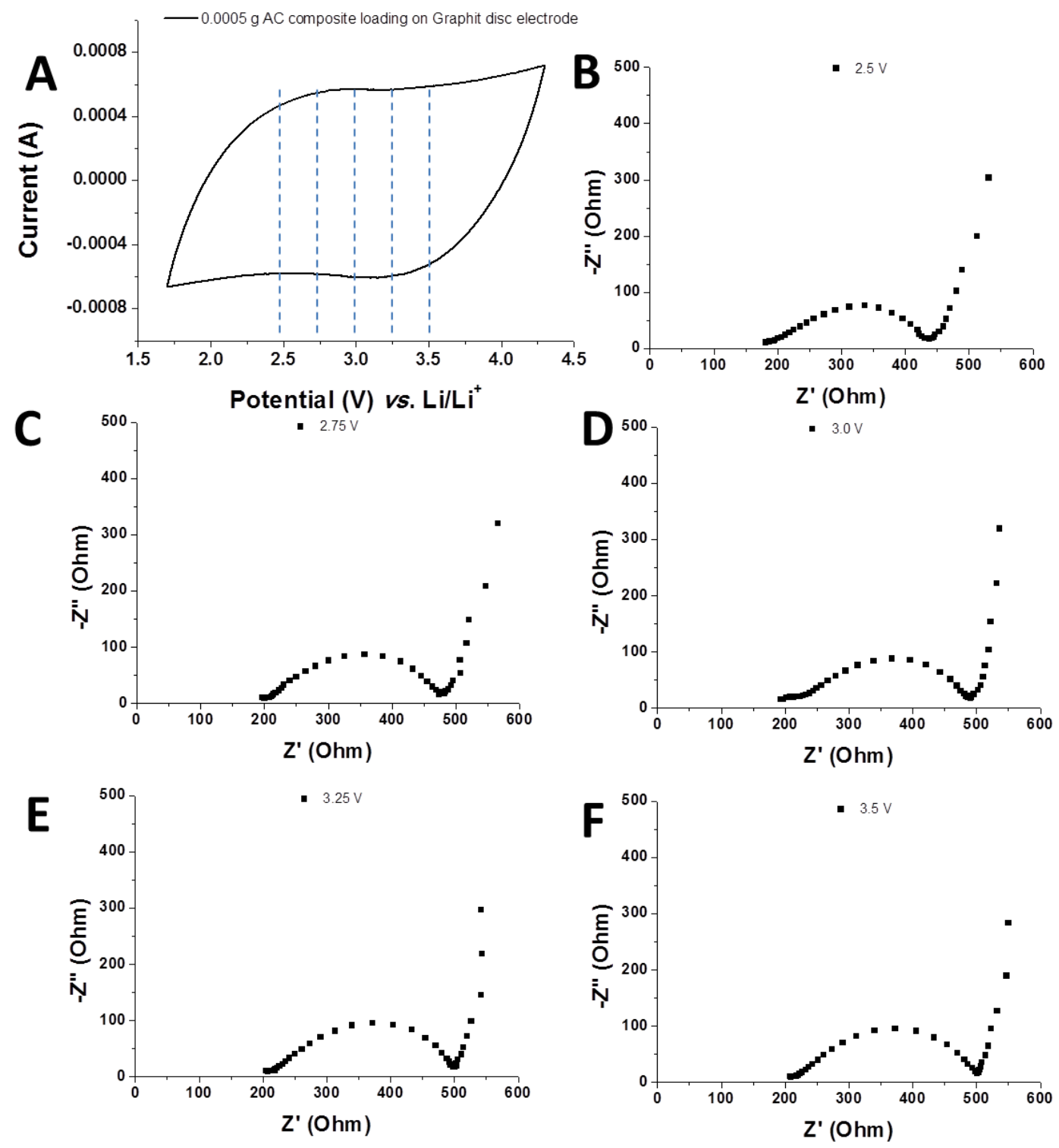

4 Fig. 4 (A) $\mathrm{CV}$ of $0.5 \mathrm{mg}$ Kuraray Act-C composite (containing $5 \% \mathrm{w}$. PVDF) on a $5 \mathrm{~mm}$

5 diameter Graphite disc electrode in mixture of BMPyrrFAP and $\gamma$-GBL $(\mathrm{v} / \mathrm{v}=1 / 1)$ containing

$6 \quad 0.5 \mathrm{~mol} \mathrm{~L}^{-1} \mathrm{LiClO}_{4}$. Scan rate: $10 \mathrm{mV} \mathrm{s}^{-1}$. Nyquist plots of the same cell at the potential of (B)

$72.50 \mathrm{~V}$, (C) $2.75 \mathrm{~V}$, (D) $3.00 \mathrm{~V}$, (E) $3.25 \mathrm{~V}$, (F) $3.50 \mathrm{~V}$ vs $\mathrm{Li} / \mathrm{Li}^{+}$. A Lithium foil was used as the

8 reference and counter electrodes. Frequent range: $10 \mathrm{kHz} \sim 0.01 \mathrm{~Hz}$. 


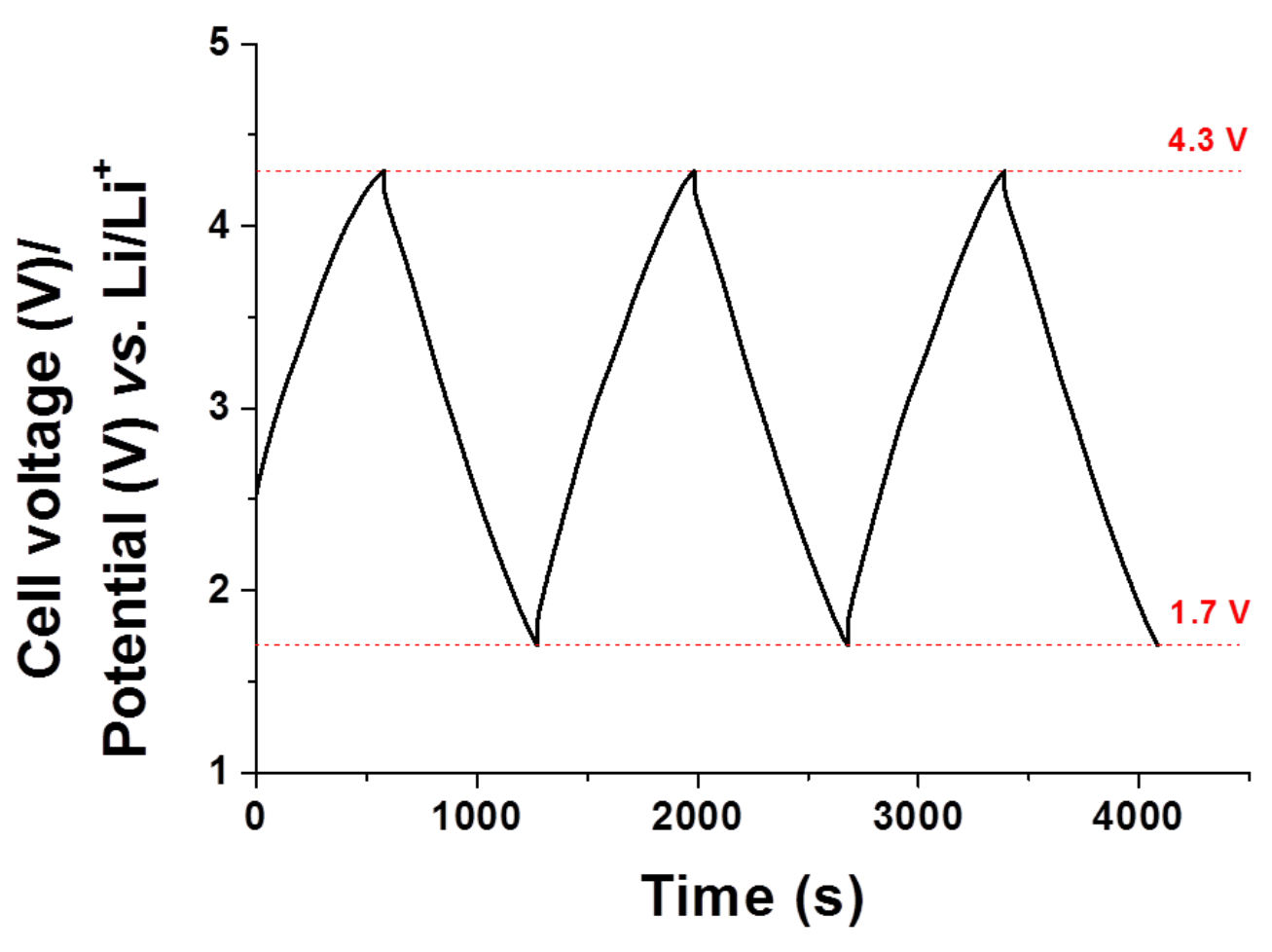

3 Fig. 5 Galvanostatic charge-discharge curves of a pellet of 0. $5 \mathrm{mg}$ Kuraray Act-C composite 4 (containing $5 \% \mathrm{w}$. PVDF) on a $5 \mathrm{~mm}$ diameter graphite disc electrode in mixture of 5 BMPyrrFAP and $\gamma$-GBL $(\mathrm{v} / \mathrm{v}=1 / 1)$ containing $0.5 \mathrm{~mol} \mathrm{~L}^{-1} \mathrm{LiClO}_{4}$. Current density: $1 \mathrm{~mA} \mathrm{~cm}^{-2}$.

The galvanostatic charge-discharge test was run under a current density of $1.02 \mathrm{~mA} \mathrm{~cm}$.

8 Because the negative electrode is a lithium foil, the voltage of the cell decays smoothly and 9 linearly during the discharging process from 4.3 to $1.7 \mathrm{~V}$. Because the charging and discharging 10 curve is almost symmetric, we still can calculate the specific capacitance and energy capacity by 11 the method for supercapacitor. The Act-C specific capacitance value comes to $107 \mathrm{~F} \mathrm{~g}^{-1}$ in the 12 ionic liquid solution containing $\mathrm{LiClO}_{4}$. Noted that only tiny amount of lithium metal takes part 13 in the charge transfer during the charging and discharging process and the molecular weight of 
1 Lithium is only $6.94 \mathrm{~g} \mathrm{~mol}^{-1}$, we can ignore the mass of the negative electrode if the active

2 materials are counted only in the calculation of specific energy capacity. The value is $232 \mathrm{Wh}$

$3 \mathrm{~kg}^{-1}$.

4 If we only chose an ionic liquid as the electrolyte, we can hardly use the available redox

5 couple resources to store charge in the energy storage devices. The Li-ion source ionic liquids

6 electrolyte can both supply sufficient charge carrier for the charge storage on the Act-C surface

7 and the necessary Li-ion for the chemical reaction. We do notice that the $\mathrm{CV}$ of the Act-C in a

8 pure ionic liquid show partly pseudo-capacitance behaviour. This is a preliminary proof for the

9 charge transfer or charge isolation process on the carbon surface. The advantage of the organic

10 electrolytes or ionic liquid electrolytes is that they can keep chemical and thermal stable in a

11 wider potential or temperature range than the one of aqueous electrolyte. Although recent studies

12 have revealed that some aqueous electrolyte can be superior to its analogous species on voltage ${ }^{8}$

13 and temperature limit, ${ }^{23}$ the variety and particularity of ionic liquids still make themselves

14 competitive in different application, especially in energy storage. This paper reveals a new idea

15 on using the Act-C materials as a positive electrode material in supercapattery. The following-up

16 work on further understanding the charge storage mechanism of Act-C in ionic liquids and

17 optimization of the hybrid design of the supercapattery in this paper is ongoing.

19 Conclusions

20 A hybrid supercapattery design comprising a lithium metal negative electrode and an

21 activated carbon positive electrode is presented and demonstrated using an experimental cell in

22 this paper. An ionic liquid was used as one component of the electrolyte containing $\mathrm{LiClO}_{4}$. The

23 Kuraray activated carbon shows both EDLC and pseudo-capacitor behaviour in a solo ionic 
1 liquid electrolyte. In the ionic liquid solution, the sufficient $\mathrm{Li}$-ion in the ionic liquid electrolyte

2 provide additional charge carriers and enough charge storage species through the $\mathrm{Li} / \mathrm{Li}^{+}$redox

3 reaction. The energy capacity of the demonstrative supercapattery reaches to $232 \mathrm{Wh} \mathrm{kg}^{-1}$, while

4 the device can present capacitor-like behaviour when the Galvanostatic charge-discharge current

5 density is more than $1.0 \mathrm{~mA} \mathrm{~cm}{ }^{-2}$, maintaining its high power output character. In addition, the

6 partly pseudo-capacitor behaviour of the activated carbon in ionic liquid reveal a possibility to

7 study the interaction of redox species inside the micropore structure of the activated carbon

8 materials.

9

10 Acknowledgements

11 This work received funding from the University of Nottingham Ningbo China and Ningbo

12 Municipal Government (3315 Plan and IAMET Special Fund, 2014A35001-1). Responsibility

13 for the content of this paper lies with the authors.

14

15 Notes and references

16 1. A. J. Stevenson, D. G. Gromadskyi, D. Hu, J. H. Chae, L. Guan, L. P. Yu and G. Z. Chen, in 17 Nanocarbons for Advanced Energy Storage, Vol. 1, ed. X. Feng, Wiley-VCH, Weinheim, $18 \quad 2015,179-210$.

19 2. S. W. Woo, K. Dokko, H. Nakano and K. Kanamura, Electrochemistry, 2007, 75, 635-640.

20 3. A. Krause, P. Kossyrev, M. Oljaca, S. Passerini, M. Winter and A. Balducci, J. Power $21 \quad$ Sources, 2011, 196, 8836-8842.

22 4. K. C. Ng, S. W. Zhang, C. Peng and G. Z. Chen, J. Electrochem. Soc., 2009, 156, A846$23 \quad$ A853. 
1 5. S. W. Zhang, C. Peng, K. C. Ng and G. Z. Chen, Electrochim. Acta, 2010, 55, 7447-7453.

2 6. Z. Dai, C. Peng, J. H. Chae, K. C. Ng and G. Z. Chen, Sci. Rep., 2015, 5, 09854.

3 7. C. Peng, S. Zhang, X. Zhou and G. Z. Chen, Chem. Comm., 2010, 3, 1499-1502.

4 8. J. H. Chae and G. Z. Chen, Electrochim. Acta, 2012, 86, 248-254.

5 9. X. Zhou, C. Peng and G. Z. Chen, AIChE J., 2012, 58, 974-983.

6 10. S. Makino, Y. Shinohara, T. Ban, W. Shimizu, K. Takahashi, N. Imanishi and W. Sugimoto, $7 \quad R S C A d v ., 2012,2,12144-12147$.

8 11. P. Wasserscheid and T. Welton (Eds.), Ionic Liquids in Synthesis, Wiley-VCH Verlag 9 Weinheim/Germany, 2002.

10 12. M. Oromi-Farrus, J. Eras, G. Villorbina, M. Torres, V. Llopis-Mestre, T. Welton and R. 11 Canela, Anal. Sci., 2008, 24, 1341-1345.

12 13. L. Chen, Y. Chi, X. Zheng, Y. Zhang and G. Chen, Anal. Chem., 2009, 81, 2394-2398.

13 14. T. Li, B. Li, S. Dong and E. Wang, Chem. Eur. J., 2007, 13, 8516-8521.

14 15. X. J. Wei, L. P. Yu, D. H. Wang, X. B. Jin and G. Z. Chen, Green Chem., 2008, 10, 296-305.

15 16. X. J. Wei, L. P. Yu, X. B. Jin, D. H. Wang and G. Z. Chen, Adv. Mater., 2009, 21, 776-780.

16 17. L. P. Yu and G. Z. Chen, RSC Adv., 2014, 4, 40281-40285.

17 18. M. Anouti, E. Couadou, L. Timperman and H. Galiano, Electrochim. Acta, 2012, 64, 110$18 \quad 117$.

19 19. A. Orita, K. Kamijima and M. Yoshida, J. Power Sources, 2010, 195, 7471-7479.

20 20. M. Galinski and I. Stepniak, J. Appl. Electrochem., 2009, 39, 1949-1953.

21 21. D. G. Gromadskyi, J. H. Chae, S. A. Norman and G. Z. Chen, Appl. Energ., 2015, 159, 3922 50. 
1 22. L. P. Yu, H.J. Sun, J. He, D. H. Wang, X. B. Jin, X. H. Hu and G.Z. Chen, Electrochem. 2 Commun., 2007, 9, 1374-1381.

3 23. Y. Gao, Z. Qin, L. Guan, X. Wang and G. Z. Chen, Chem. Commun., 2015, 51, 10819$4 \quad 10822$. 